\title{
Color Analysis on business suit and its application research
}

\author{
${ }^{1 \mathrm{a}}$ Bian Fei ${ }^{2} \mathrm{Ni}$ Ming ${ }^{3}$ Zong Minying \\ Donghua University in No. 1882, Yan'an West Road, Shanghai city \\ aEmail:bianfei526@163.com
}

Corresponding author: Liu Canming

Key words: business suit design; color design; business suit; color psychology

\begin{abstract}
Business suit refers to the community uniforms in workplace, the business suit design is the design created for uniforms. It is both the occupational service refers to the group of clothing for the workplace,
\end{abstract}

Occupational service design is for the professional service and the design of the system to create, but more is a social things. This design system is not just a single uniform, but also involves the scope that working suit can impact-the impact on the overall image of the enterprise and workers' emotion, even the industry trend of the entire working suit.

Viewing from the perceptual factors of the suit, the art of the design of working unit is to take into consideration the modeling, color, fabric, technology and population that constitute the garment as well as beauty-appreciation, and beautify the form of clothing with clothing and accessories while highlighting the characteristics of corporate image. Attention need to be particularly paid to the impact that working suit have on peoples psychology. The color of the working suit that suite the working environment can make people happy and improve work efficiency.

With the development of economic globalization and the advance of market economy, all walks of life are increasingly focused on the shaping of the employee's image in order to achieve their own development and foster their own brand culture and corporate image. Looking through the successful enterprise both at home and abroad, there is no one without their own iconic design on professional garments, so the employees of the enterprises play an indispensable role between industries. 


\section{The psychological association of the color of professional garments}

The psychological association of professional garments color refers to the psychological response of people on the color of professional garments, which is also the peoples visual function and feel. Long-term natural life and social life experience allow people to make different judgment on the psychological value and performance value that each color has. From a certain point of view, different colors exert different psychological associations on peoples mind. Such as color can give people the feeling of excitement or quiet, such feeling will give people positive or negative emotions in different working environment because it is related to the hue, brightness and purity of color., red, yellow, Orange and other colors make people feel excited, representing health, warmth, happy. Blue and cyan make people feel calm, standing for mature and delicate. Another example is that color can not only give people a magnificent sense of beauty, but also may give a sense of simplicity. In general, rich bright color displays a beautiful and pure feeling, gray and dark color gives a natural beauty sense. Warm color and color in high purity are lively and active while cool color and color in low purity give a solemn sense. Generally lively color is chosen for school uniform to show their vibrant and youth while the solemn uniform is suitable for management staff to show the maturity of the dresser (Picture 1-1).

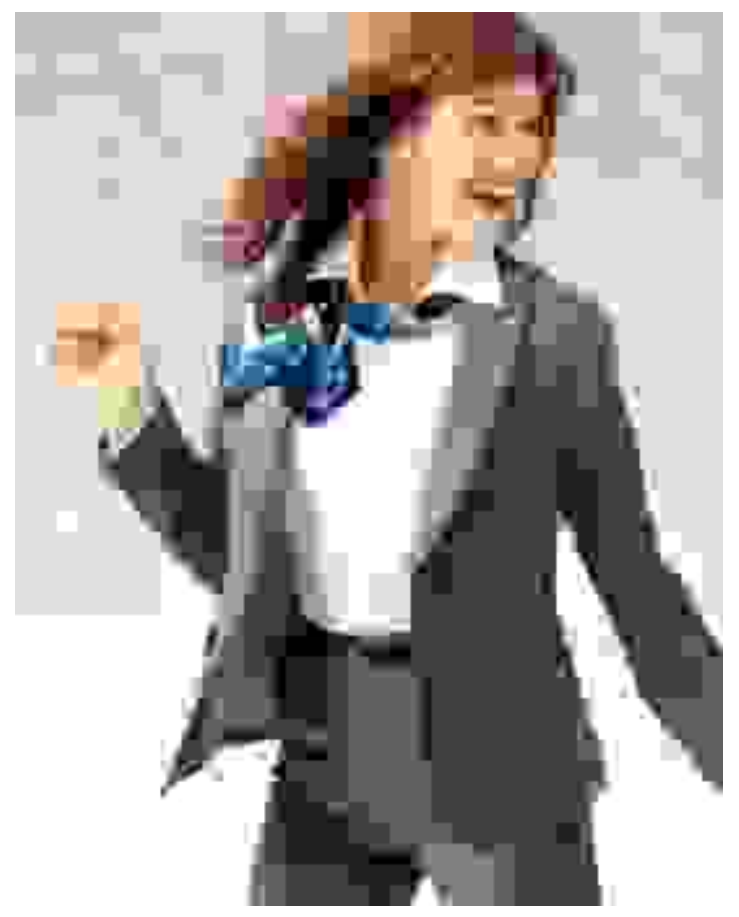

Picture 1-1 Workplace management and the civil personnel's apparel 


\section{The selection basis of the color of professional garments}

\section{(1) Corporate identity system}

Corporate identity system is used by the enterprise to propagate its business philosophy, corporate culture and business activities to shape the corporate image through a unified visual design and using the overall communication system, so that it is a strategic activities and functions that arouse a sense of identity and common values among the public. For the enterprise that introducing the uniform designed by the corporate identity system, a cohesion and image can be formed from inside to outside. From the internal, all staff dressed in unified uniform identifying clear position to work regularly without confusion to gradually form centripetal force and cohesion, which is conducive to stimulate each employee to work hard in accordance with the concept and spirit of the enterprise; from the outside, users, consumers and visitors will have good impression and value identity through the professional garment, related product and service quality, which in turn increasing the competitive advantage of the enterprise.

Furthermore, the company's iconic color has been widely used in the enterprise logo, advertising, slogans, trademarks, flags, construction and other aspects. With the popularity and deepening of the concept of visual recognition and the improvement of the probability of product homogenization, it is more and more difficult to identify an enterprise with color, so when choosing color for the professional garment, you can give preference to the iconic color and use it as the main color of staffs uniform and match it with other colors to highlight the enterprises individualized packaging to perfect the corporate identity system. Such as the EMS Express that we are very familiar with, the wholly-owned company under the China Post Group, mainly engage in international and domestic EMS business. It is China's first supplier of courier services and also the largest operator and leader in the Express Industry in China. The postal green and the enterprises iconic blue, orange and white have been widely used in employee uniforms, envelopes, advertisements, websites, etc, forming a complete corporate identity system. The couriers wearing uniform are like a live advertisement facilitating the awareness of the brand. Therefore, the enterprise identity system is the overall orientation of the choice of color for professional garment.

\section{(II) Sense of integration}

Professional garment is different from the general clothing because which occasion is suitable for the general clothing is determined by the purchaser but the professional garment is inseparable to its 
dresser group and occasions. The color of all the uniform of the enterprise must be provided with a sense of series and in harmony with the dressers working environment. Successful uniform color often requires the designer with a keen sense on matching and accurately grasps various attempts. When designing a uniform or a serious of uniform, the designer takes the occasion, season and color matching rules into consideration in choosing the color, tone and color matching in order to reflect the integration of the color design of uniform. Take the costume for medal ceremony of 2008 Beijing Olympic Games as an example, womens wear of fifteen style is divided into five series, series one: the design aspiration of blue and white porcelain series are from the world famous Chinese blue and white porcelain; series two: sapphire blue series adopt gentle and elegant royal blue as the main color of the dress; series three: satin dress in Chinese scholar tree green mean vibrant life and lush environment and embody the good wishes of harmonious development of nature and man and the strong determination to Green Olympics; series four: the jade white cleverly echoed the concept of inlaying jade in the Olympic medal; series five: for the pink series, that waist decorations in Po-phase flowers pattern produced by traditional embroidery craft segment out the perfect proportion of the human body. The color of the dress of each series is coordinated with the image landscape of the Beijing Olympic Games and the award ceremony environment, highlighting the Chinese elements, national characteristics and time feature in the technique of expression and leaving a valuable cultural heritage. Therefore, integration is the detail need in choosing the color of uniform.

\section{(III) Safe operation}

Some profession's working need determines the color selection of its uniform, the color of these uniforms is not selected randomly but taking the workers safe operation and work environment into consideration and according to the exact environment and experience and general knowledge, so the application of color of professional garment has become an international practice. For example, orange for the rescue workers uniform is particularly eye-catching in the ruins after the earthquake and other disasters, which facilitate the staff and trapped people to find each other; the medical workers of each hospital operating room are wearing green surgery clothes, this is the measures taken to eliminate the effect caused by the visual complementarity of eye. When viewing a color for a long time, peoples optic nerve can be irritated easily and become fatigue, In order to reduce this fatigue, the optic nerve will induce a complementary color called self-regulation. For example, if 
you stare at a table drawn in bright color in white paper and then turn to anther blank paper, you will find the same table appears on this white paper but its color becomes light green. Doctors always see bright red blood in the course of surgery, over time, they occasionally transferred their sight to the white gown of their companions and will see the blood stains that will confuse the vision and affect the surgical results. The adopting light color fabric for the surgical gown can eliminate the green illusion and relax doctors eye, so as to ensure the smooth operation. So safe operation is the restrictions for the selection of color for some professional garment.

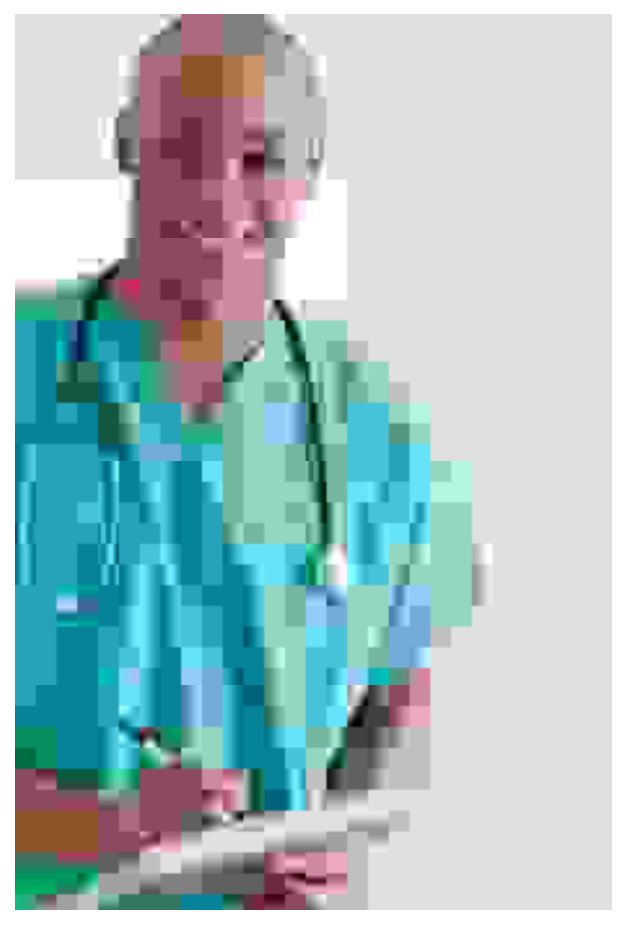

Picture 1-2 Doctors' surgical gown

\section{(IV) Detail differentiation}

Some large-scale enterprises and factories have more departments and plant and each plant is responsible for a production process. For example, power, equipment, energy, casting, machine construction and other plants will be divided in some of the power tool in the north of China, so it is unreasonable to subdivide the workers professional garment of each plant from style because one is that the change of the style means the change of detail, it is difficult to quickly identify which workshop the worker belong to from long distance, second is so many styles also increase the cost of professional garment, which is unnecessary expenses. The designer can choose different color based on the management of the enterprise and the characteristics of each plant to allow the uniform of each plant has their own color, which is much easy for distinction. Therefore, detail 
differentiation is the industry need to choosing color for professional garment.

\section{(IV) Industry tradition}

Long-term industry experience accumulation and practitioner's similar psychological accumulation establish an international link between the color some of industry professional garments and the industry. The traditional color of the industry is the result of years accumulation and has strong implication in people consciousness and become one of the basic characteristics that the outside distinguishing the type of the industry. For example, the medical staff wearing white uniform gives people a quiet feeling and also makes the patient feel safe and reliable; the police wearing blue uniform bring a majestic and calm feeling to people; the chef's white uniform provides a clean dining experience and so on. This professional garments color is blessed with abstract symbolic features and strict inheritance, forming the international practice. Therefore, the designer not only need to consider the work environment, corporate logo and other objective conditions in designing professional garment, but also need to consider the long-term awareness of people. Of course, there are individual companies pursing unique and doing opposite. However, whether the traditional industry color is extended or subverted, designers should make targeted treatment based on the business requirements.

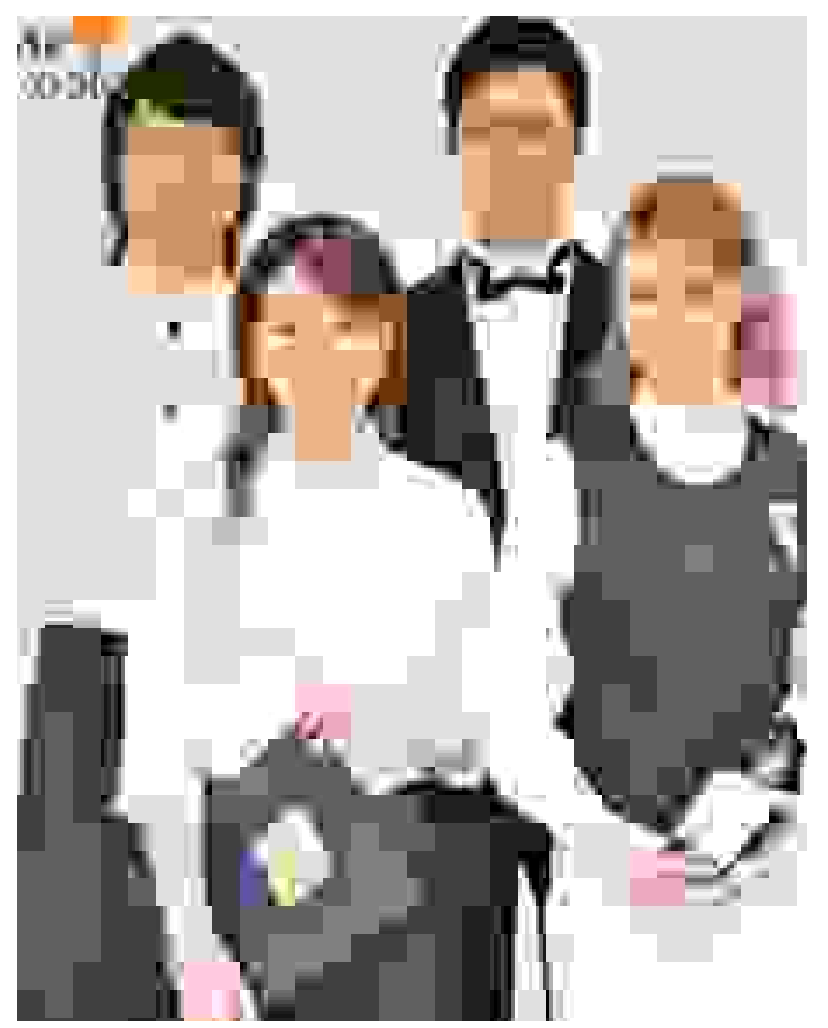

Picture 1-3 The industry's distinctive professional garments 


\section{Conclusions}

1) The implementation of professional garments is to turn the artistry of clothing into reality. It is a link that integrates the artistry of professional garment with the practical effects, achieving the perfect combination of clothing artistry with the real benefits. It is found through the observation and study on the object, place, purpose, occupation, psychological and physiological demand of the professional garment that color may be more stimulated to people senses than graph and text.

2) the color of professional garment is an important link of the enterprise's basic design project. Through the design and selection of the color of the professional garment, the enterprise can both create a good image and lay a basis for its marketing strategy.

Acknowledgements: Supported by Shanghai IV Summit Discipline in Design--- Fashion design and brand image transmission

\section{References}

[1] Color design analysis of professional garment Liu Kun Modern commerce industry 06 phase 2012

[2] The analysis and application of the color design of Chinese occupational apparel He Jun Zhang Yiyi Shandong Textile Economy 01 phase 2015

[3] Analysis on the color psychology of professional garment for different position level Chai Lifang Popular Science \& Technology 04 phase 2008

[4] The application and research of blue emotional factors in professional garment Li Qiong Hunan University of Technology Master's thesis

[5] Zou You professional garment design. Beijing China TeXtile Press, February 2007

[6] Chang Shuxiong Wang Xiaoying Professional garment design tutorials. Liaoning. Liaoning Fine Arts Publishing House, June 2014 